\title{
Hypoxic Condition Up-Regulates the Expression of Angiopoietin-2 in ADSCs
}

\author{
Lingxiao Ye, Xiaoming Sun, Yuguang Zhang*, Ying Zhang*
}

Department of Plastic and Reconstructive Surgery, The Ninth People's Hospital Affiliated to School of Medicine Shanghai Jiao Tong University, Shanghai, China

Correspondence to: Yuguang Zhang, zyinghh@163.com; Ying Zhang, zyinghh@163.com

Keywords: ADSCs, ANGPT2, Angiogenesis, Wound Healing

Received: January 10, $2019 \quad$ Accepted: February 18, $2019 \quad$ Published: February 21, 2019

Copyright $\odot 2019$ by authors and Scientific Research Publishing Inc.

This work is licensed under the Creative Commons Attribution International License (CC BY 4.0).

http://creativecommons.org/licenses/by/4.0/

\section{(c) (i) Open Access}

\section{ABSTRACT}

Wound healing requires abundant nutrition and blood supply, thus angiogenesis is a vital stage in this process. Angiogenesis involves diverse kinds of immune cells, growth factors, cytokines and inhibitors. ADSCs, especially ADSCs cultured in hypoxic condition, are reported to be able to facilitate angiogenesis and promote wound healing process. Significant efforts have been made on the development of ADSCs-based therapies with wound-healing applications. Here the results showed that expression of Angiopoietin-2 (ANGPT2) in ADSCs was up-regulated in the hypoxic condition.

\section{INTRODUCTION}

Wound healing remains a central concern for plastic surgeons. When traumatic injury happens to the healthy skins, the normal anatomical structure of the local area would be destroyed. Wound healing could be influenced by many factors such as nutritional status of the host and the local area, infections, inflammatory response, proteases as well as stem cells. Recent researches suggested that the local wound environment could be the key target for therapeutic strategies [1].

Blood vessel growth is a crucial point of wound healing, as vessels provide essential nutrition and oxygen at the wound site for tissues and cells. Angiogenesis plays an important part in wound healing procedure [2]. Inadequate angiogenesis wound weaken the repair efficiency of wound and finally rise up to poor healing [3].

Adipose-derived stem cells (ADSCs) were reported to be effective in angiogenesis and wound healing process. Stem-cell therapy strategies have raised more and more attention by surgeons [4]. Though studies much, the mechanism of ADSCs involving the wound healing process remained inconclusive.

Previous articles have reported that endothelial cell-derived angiopoietin-2 (ANGPT-2) could control the angiogenesis and regeneration of liver [5]. In this article, authors wanted to explore the role of ANGPT-2 as well as ADSCs in the wound healing procedure. Meanwhile authors aimed to explore the relationship between hypoxic condition and the expression of ANGPT-2 in ADSCs. 


\section{MATERIALS AND METHODS}

\subsection{Cell Culture}

\section{Isolation and culture of ADSCs}

Liposuction aspirates were harvested from the abdomen of a plastic surgery patient. Liposuction aspirates was transported and washed with phosphate buffer saline(PBS) and digested with $1 \mathrm{mg} / \mathrm{ml}$ type I collagenase for $40 \mathrm{~min}$ at $37^{\circ} \mathrm{C}$ with shaking, filtered through a $140 \mu \mathrm{m}$ nylon mesh and centrifuged at $1500 \mathrm{r} / \mathrm{min}$ for $5 \mathrm{~min}$ at room temperature (RT). The digested tissue was filtered through gauze to separate it from the undigested tissue and centrifuged at $1500 \mathrm{r} / \mathrm{min}$ for $5 \mathrm{~min}$ at RT. The supernatant (floating adipocytes) was discarded. The pellet (ADSC fraction) was resuspended in the medium (1\% penicillin/streptomycin + DMEM/Low glucose $+10 \% \mathrm{FBS})$ and transferred into culture dishes. Here we obtained the primary ADSCs.

ADSCs were divided into two groups: ADSCs cultured in normal oxygen concentration (AN group), ADSCs cultured in low oxygen concentration (AL group). AN group were cultured separately at $37^{\circ} \mathrm{C}$ in a normoxic $\left(20 \% \mathrm{O}_{2}, 5 \% \mathrm{CO}_{2}\right)$ condition and AL group in hypoxic $\left(5 \% \mathrm{O}_{2}, 5 \% \mathrm{CO}_{2}\right)$ with medium changes every 3 days. When the cells reached approximately $80 \%$ confluence, subculture (passage) was performed. ADSCs at passages P3-P5 were used for the studies.

\subsection{Morphology of ADSCs in Different Oxygen Environment}

To examine possible morphological difference between AN group and AL group, we evaluated their morphological changes by optics microscope in different magnifications at passage 3. Images were recorded by the computer.

\subsection{Expression of ANGPT-2 for ADSCs by Immunofluorescent Staining}

Two groups of ADSCs and five groups of ADSCs were seeded into 6-well plates. There was a glass slide on the bottom in each plate to load cells. When the cells reached approximately $70 \%$ confluence, the cells were fixed with 4\% PFA for $20 \mathrm{~min}$ at RT and embedded with paraffin for further analysis. The following primary antibodies were used in this study: goat anti mouse RedX (1:400, Shanghai Daixuan biotechnology), Goat anti rabbit FITC (1:400, Shanghai Daixuan biotechnology), DAPI (1:500, Shanghai Daixuan biotechnology). After incubation with fluorophore-conjugated secondary antibodies, samples were further stained with DAPI. Images were taken with the Olympus CX53 inverted fluorescence microscope (Nikon, Japan).

\subsection{Human Angiopoietin-2 ELISA Kit}

The supernate of cultured cells (including 7 groups of cells as referred before) was collected into 1-ml epoxide tubes and stored in $-80^{\circ} \mathrm{C}$ refrigerator for further test. In this research, authors used human ANGPT-2 ELISA Kit (EK0657, Shanghai Daixuan biotechnology) to detect the content of ANGPT-2 in the supernate of cultured cells.

\subsection{Real-Time Quantitative RT-PCR for ADSCs}

Quantification was performed with a two-step reaction process: reverse transcription (RT) and PCR. Each RT reaction has two steps. The first step is $0.5 \mu \mathrm{g}$ RNA, $2 \mu \mathrm{l}$ of $4 \times$ gDNA wiper Mix, add Nuclease-free $\mathrm{H}_{2} \mathrm{O}$ to $8 \mu \mathrm{l}$. Reactions were performed in a GeneAmp ${ }^{\circledR}$ PCR System 9700 (Applied Biosystems, USA) for $2 \mathrm{~min}$ at $42^{\circ} \mathrm{C}$. The second step is adding $2 \mu \mathrm{l}$ of $5 \times$ HiScript II Q RT SuperMix IIa. Reactions were performed in a GeneAmp ${ }^{\circledR}$ PCR System 9700 (Applied Biosystems, USA) for $10 \mathrm{~min}$ at $25^{\circ} \mathrm{C} ; 30 \mathrm{~min}$ at $50^{\circ} \mathrm{C} ; 5 \mathrm{~min}$ at $85^{\circ} \mathrm{C}$. The $10 \mu \mathrm{RT}$ reaction mix was then diluted $\times 10$ in nuclease-free water and held at $-20^{\circ}$ C. Real-time PCR was performed using LightCycler ${ }^{\circledR} 480$ II Real-time PCR Instrument (Roche, Swiss) with $10 \mu \mathrm{l}$ PCR reaction mixture that included $1 \mu \mathrm{l}$ of cDNA, $5 \mu \mathrm{l}$ of $2 \times$ QuantiFast $^{\circledR}$ SYBR $^{\circledR}$ Green PCR 
Master Mix (Qiagen, Germany), $0.2 \mu \mathrm{l}$ of forward primer, $0.2 \mu \mathrm{l}$ of reverse primer and $3.6 \mu \mathrm{l}$ of nuclease-free water. Reactions were incubated in a 384-well optical plate (Roche, Swiss) at $95^{\circ} \mathrm{C}$ for $5 \mathrm{~min}$, followed by 40 cycles of $95^{\circ} \mathrm{C}$ for $10 \mathrm{~s}, 60^{\circ} \mathrm{C}$ for $30 \mathrm{~s}$. Each sample was run in triplicate for analysis. At the end of the PCR cycles, melting curve analysis was performed to validate the specific generation of the expected PCR product. The primer sequences were designed in the laboratory and synthesized by Generay Biotech (Generay, PRC).

The expression levels of mRNAs were normalized to ACTB and were calculated using the $2^{-\Delta \Delta C t}$ method.

\subsection{Microarray Analysis for ADSCs}

Microarrays were performed by the core facility of the Shanghai OE biotech Co. Ltd. In brief, total RNA was extracted from freshly isolated ADSCs using the RNeasy kit (Qiagen, Germany). RNA was eluted in RNAase free water. The quality and concentration of total RNA was checked by gel analysis using the total RNA Nanochip assay on an Agilent 2100 Bioanalyzer and NanoDrop. The samples were then reverse transcribed into biotin labeled cDNA and hybridized according the manufacture's instructions. Microarray data were collected and analyzed with Agilent microarray scan platform.

\section{RESULTS}

\subsection{Morphology of AL Group and AN Group}

As is demonstrated in Figure 1, Cells of AL showed a more loose-bodied morphology. The content of

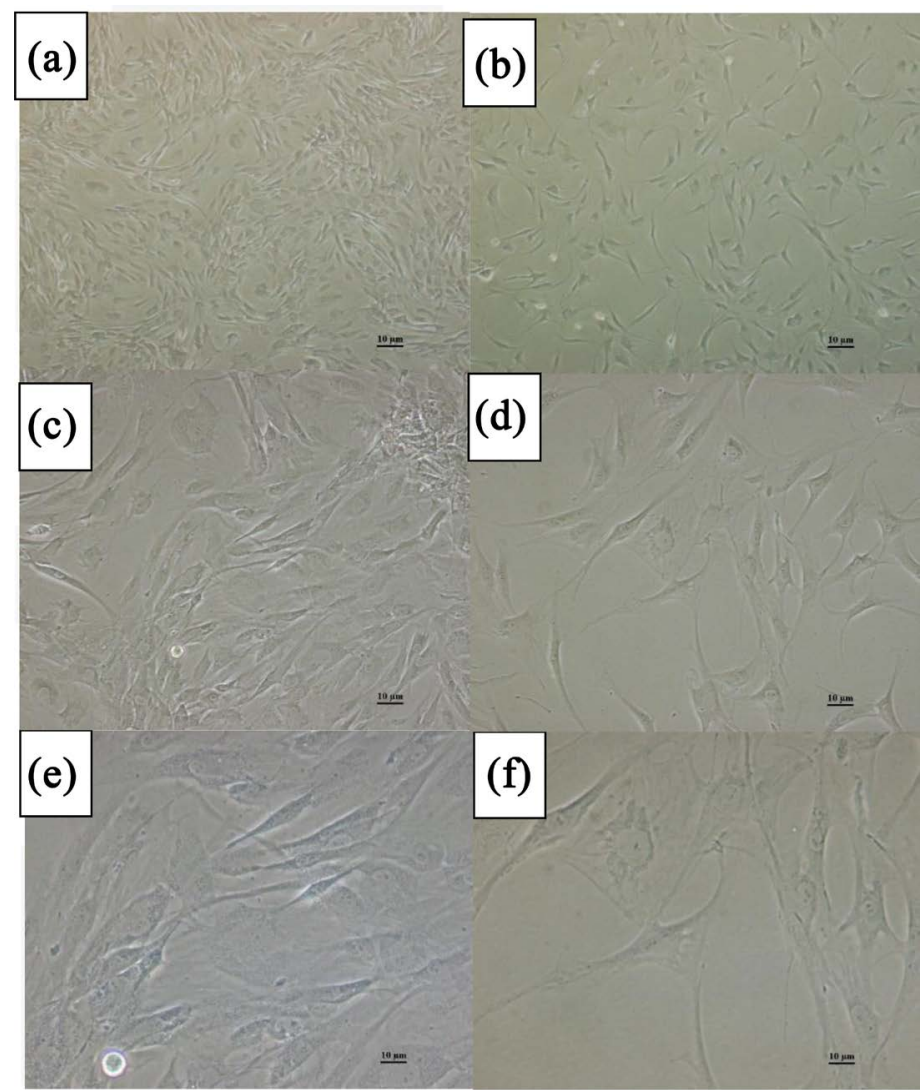

Figure 1. Difference between the AL group (left) AN group (right) could be observed. the Morphology of AL group maninified at $40 \times(\mathrm{a}), 100 \times(\mathrm{c}), 200 \times$ (e) and $\mathrm{AN}$ group at $40 \times(\mathrm{b}), 100 \times(\mathrm{d}), 200$ $\times(e)$ are exhibited in Figure 4. 
cytoplasm turned out to be increased.

\subsection{Results of Immunofluorescent Staining}

ADSCs cultured in normoxic condition exhibited weaker fluorescence reaction than ADSCs cultured in hypoxic condition, as is demonstrate in Figure 2. The result demonstrated the ADSCs cultured in hypoxic wound generate significantly more ANGPT-2 protein than ADSCs traditionally cultured.

\subsection{The ANGPT-2 Concentration ANALYSIS}

The ANGPT-2 concentrations in supernate of cells were quantified by ELISA. The average ANGPT-2 concentration in the supernate of AL group was $267.453 \mathrm{pg} / \mathrm{ml}$ which was significantly higher than that in the supernate of AN group as is showed in Table 1.

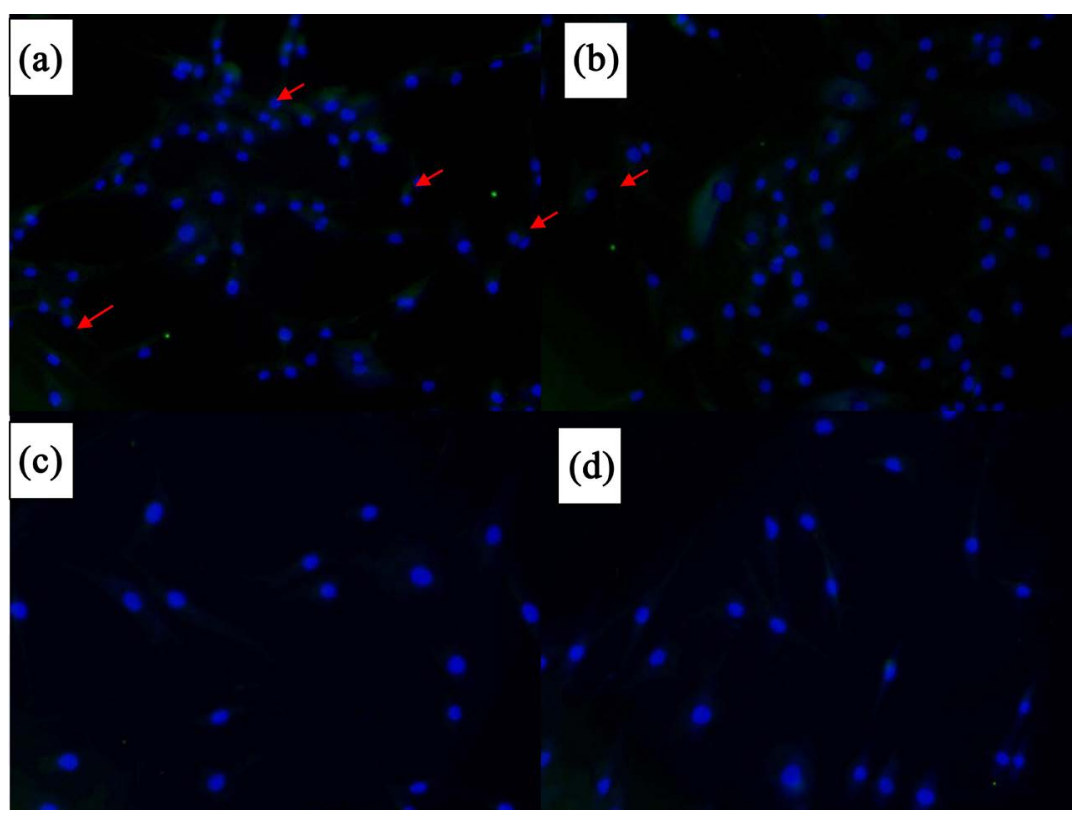

Figure 2. The nucleus was stained blue as showed in figure. A few fluorescence reactions could be detected (red arrow) in AL group. However, we could hardly find fluorescence reaction in AN group ((c), (d)) observed At 200 magnification.

Table 1. The reulsts of ELISA.

the concentration of ANGPT-2 for ADSCs

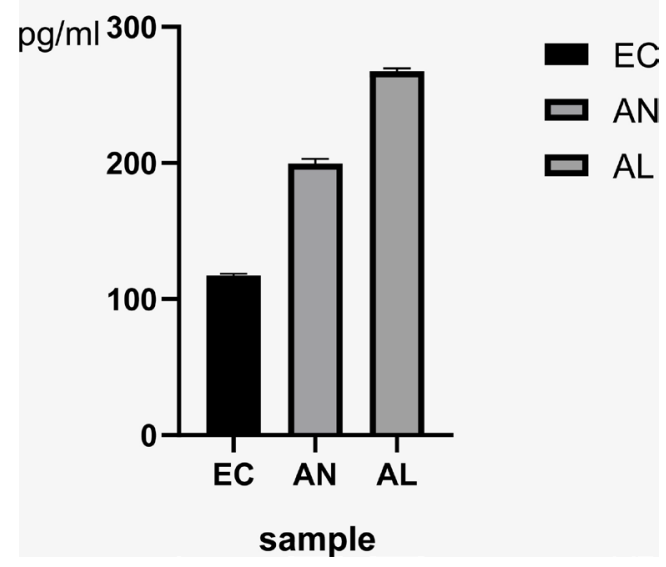




\subsection{Microarray Analysis}

The different expression level of concerning gene is demonstrated by the difference of color. We could see in Figure 3 that the expression level of ANGPT-2 showed significant difference between the AN group and the AL group. In the AL group, the expression of ANGPT-2 was significantly up-regulated as is showed. Otherwise, the gene expression of VEGFA also showed significant difference, VEGFA was apparently down-regulated in the AL group.

\subsection{Expression Level of ANGPT-2, VEGF Gene}

The level of expression was calculated by $2^{-\Delta \Delta C t}$ method.

$$
\begin{gathered}
\Delta C t=C t_{\text {target gene }}-C t_{\text {reference gene }} \\
\Delta \Delta C t=\Delta C t_{\text {sample }}-\Delta C t_{\text {control }}
\end{gathered}
$$

relative transcript level $=2^{-\Delta \Delta C t}$

As a result, compared to the AN group, the expression levels of ANGPT-2 for AL group were up-regulated about 3.45 times, and the expression levels of VEGF for AL group were down-regulated about 0.42 times as is illustrated in Figure 4.

\section{DISCUSSION}

Wound healing after skin injury could be an extremely complex event. It involves numerous communications between different cells and tissues. It demands a nutritious environment with low infectious risk. If the host fails to provide the essential blood supply, the injured skin might end up with either an ulcerative skin defect (chronic wound) or an excessive formation of scar (hypertrophic scar or keloid).

Angiogenesis is a critical step in the wound healing process: the growth of capillaries from pre-existing vasculature in the undamaged surrounding tissue [6]. This procedure also involves plenty of immune cells, growth factors, cytokines and stem cell therapies. ADSCs-based therapies have already been applies in many wound-healing cases. And ADSCs cultured in hypoxic condition wound work better in the procedure of wound healing [7]. These cells have been shown to increase neovascularization [8], reinforce

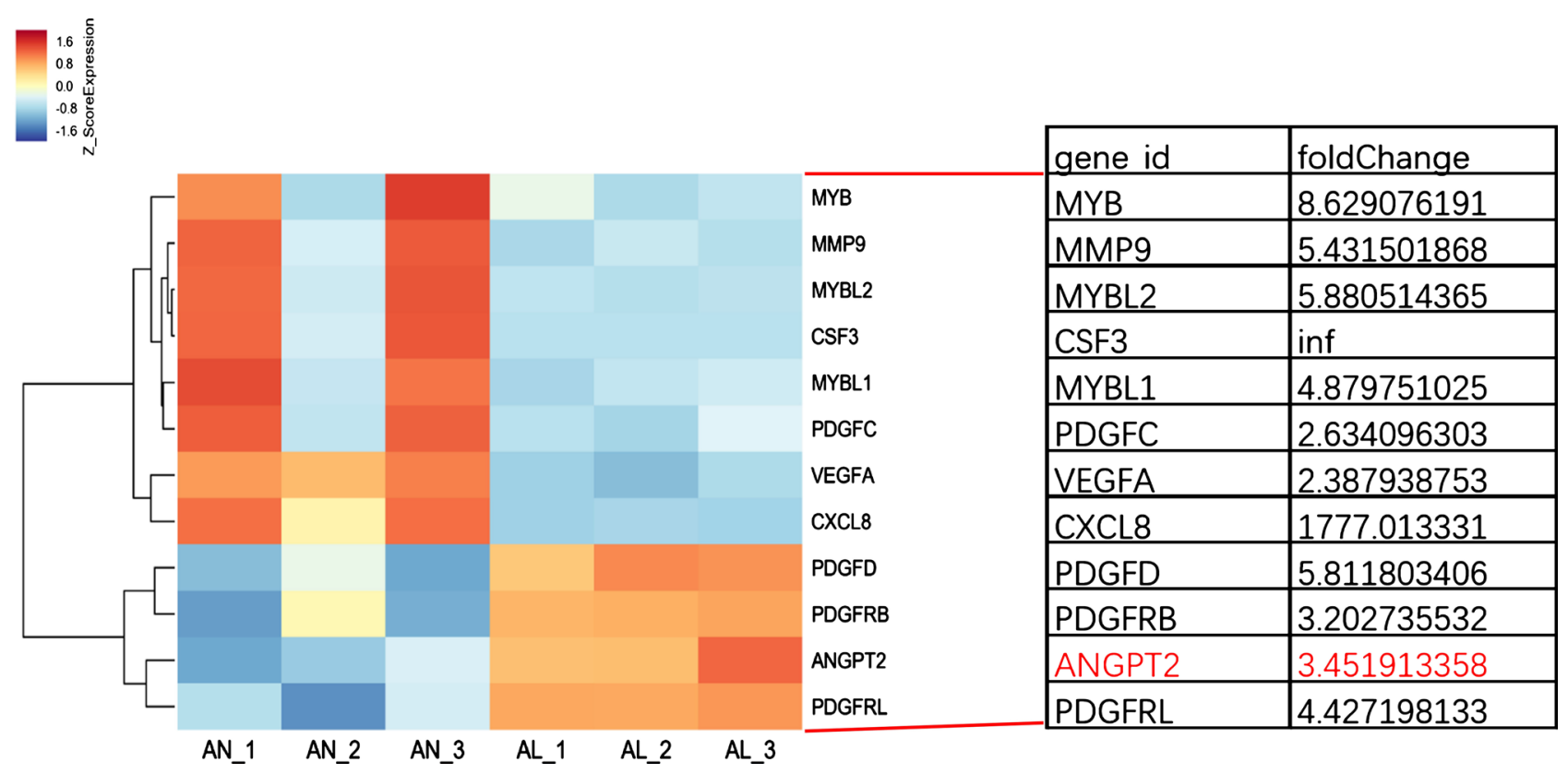

Figure 3. The results of Microarray analysis were exhibited as above. 


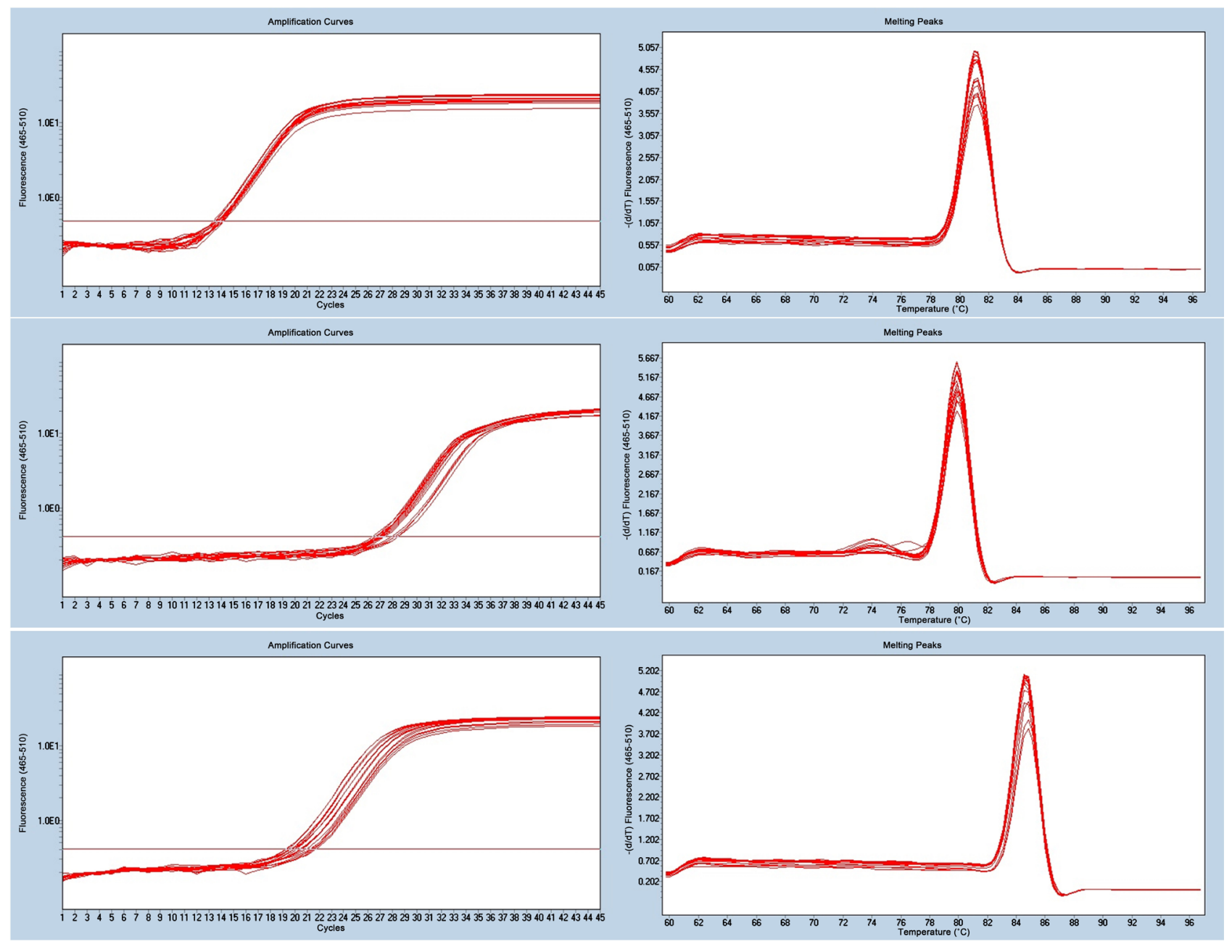

Figure 4. The Amplification Curves (upper) and Melting Peaks (lower) of ACTB (left), ANGPT-2 (middle), VEGF (right).

recruitment of other cell types to the sight of injury [9], and facilitate growth and migration among surrounding cells [4] involved in the wound-healing process. Also, It is worthy to mention that vascular endothelial growth factor (VEGF) plays an vital part especially at the wound healing procedure [10, 11].

ANGPT-2 could up-regulate the expression of VEGFR2 and could facilitate the angiogenesis process and the generation of liver [5]. However, it is none to wiser what kind of role ANGPT-2 plays in the wound healing process. The function of ANGPT-2 in the wound healing process has not been studied thoroughly yet.

Nevertheless, the accurate knowledge and mechanism about the relationship between ADSCs and ANGPT-2 in such procedure remains unrevealed.

To find further conclusions, authors cultured ADSCs under hypoxic condition in this study. Authors were aimed at exploring the changes on the expression of ANGPT-2 for ADSCs cultured in hypoxic condition. The analysis of ELISA and Immunofluorescent Staining showed consistent results with our primary suppose. The result of ELISA demonstrated the content of ANGPT-2 in the supernate of ADSCs cultured in hypoxic condition was more than that of ADSCs cultured in normoxic condition. More ANGPT-2 was secreted by ADSCs cultured in hypoxic condition.

More accurate evidences were obtained through Microarray analysis and RT-PCR in this study. Microarray analysis and RT-PCR are very sensitive analysis method. These methods could be complicated 
but they could more accurately clarify the truth. The results consistently showed there was significant difference between the content of ANGPT-2 within the ADSCs cells. The expression of ANGPT-2 in ADSCs cultured in hypoxic condition was evidently better than ADSCs cultured in normoxic condition.

To summarize, ADSCs cultured in hypoxic condition could express and secret more abundant ANGPT-2 than ADSCs cultured in normoxic condition.

\section{CONCLUSION}

As is demonstrated above, the expression of ADSCs-derived ANGPT-2 was up-regulated in hypoxic condition. This phenomenon clued us that hypoxic preconditioned ADSCs might have more potential to regulate the function of VEGF for endothelial cells and might perform a better role in the wound healing process. Further study is in sore need to illuminate the relationship between ANGPT-2 and VEGF and to illuminate their vital function in the wound healing procedure.

\section{ACKNOWLEDGEMENTS}

No outside funding was received for this article. No conflict of interest exits in the submission of this manuscript, and manuscript is approved by all authors for publication.

\section{CONFLICTS OF INTEREST}

The authors declare no conflicts of interest regarding the publication of this paper.

\section{REFERENCES}

1. Eming, S.A., Martin, P. and Tomic-Canic, M. (2014) Wound Repair and Regeneration: Mechanisms, Signaling, and Translation. Science Translational Medicine, 6, 265sr6. https://doi.org/10.1126/scitranslmed.3009337

2. Eming, S.A., Brachvogel, B., Odorisio, T. and Koch, M. (2007) Regulation of Angiogenesis: Wound Healing as a Model. Progress in Histochemistry and Cytochemistry, 42, 115-170. https://doi.org/10.1016/j.proghi.2007.06.001

3. Brem, H. and Tomic-Canic, M. (2007) Cellular and Molecular Basis of Wound Healing in Diabetes. Journal of Clinical Investigation, 117, 1219-1222. https://doi.org/10.1172/JCI32169

4. Kim, W.S., Park, B.S., Sung, J.H., Yang, J.M., Park, S.B., Kwak, S.J. and Park, J.S. (2007) Wound Healing Effect of Adipose-Derived Stem Cells: A Critical Role of Secretory Factors on Human Dermal Fibroblasts. Journal of Dermatological Science, 48, 15-24. https://doi.org/10.1016/j.jdermsci.2007.05.018

5. Hu, J.H., Srivastava, K., Wieland, M., Runge, A., Mogler, C., Besemfelder, E., Terhardt, D., Vogel, M.J., Cao, L.J., Korn, C., Bartels, S., Thomas, M. and Augustin, H.G. (2014) Endothelial Cell-Derived Angiopoietin-2 Controls Liver Regeneration as a Spatiotemporal Rheostat. Science, 343, 416-419. https://doi.org/10.1126/science.1244880

6. Bauer, S.M., Bauer, R.J. and Velazquez, O.C. (2005) Angiogenesis, Vasculogenesis, and Induction of Healing in Chronic Wounds. Vascular and Endovascular Surgery, 39, 293-306.

https://doi.org/10.1177/153857440503900401

7. Faghih, H., Javeri, A. and Taha, M.F. (2017) Impact of Early Subcultures on Stemness, Migration and Angiogenic Potential of Adipose Tissue-Derived Stem Cells and Their Resistance to in Vitro Ischemic Condition. Cytotechnology, 69, 885-900. https://doi.org/10.1007/s10616-017-0104-5

8. Cao, Y., Sun, Z., Liao, L., Meng, Y., Han, Q. and Zhao, R.C. (2005) Human Adipose Tissue-Derived Stem Cells Differentiate into Endothelial Cells in Vitro and Improve Postnatal Neovascularization in Vivo. Biochemical and Biophysical Research Communications, 332, 370-379. https://doi.org/10.1016/j.bbrc.2005.04.135

9. Gimble, J.M., Katz, A.J. and Bunnell, B.A. (2007) Adipose-Derived Stem Cells for Regenerative Medicine. Cir- 
culation Research, 100, 1249-1260. https://doi.org/10.1161/01.RES.0000265074.83288.09

10. Flegg, J.A., Menon, S.N., Maini, P.K. and McElwain, D.L. (2015) On the Mathematical Modeling of Wound Healing Angiogenesis in Skin as a Reaction-Transport Process. Frontier in Physiology, 6, 262.

11. Mantzaris, N.V., Webb, S. and Othmer, H.G. (2004) Mathematical Modeling of Tumor-Induced Angiogenesis. Journal of Mathematical Biology, 49, 111-187. https://doi.org/10.1007/s00285-003-0262-2 